BU-HEP 94-5

hep-ph/9402339

February 22, 1994

\title{
The Phenomenology of the CP-odd Scalar in Two-Doublet Models
}

\author{
Dimitris Kominis: \\ Dept. of Physics, Boston University, 590 Commonwealth Avenue, \\ Boston, MA 02215
}

\begin{abstract}
We examine the phenomenology of the $C P$-odd scalar $A^{0}$ of two-Higgs-doublet models. We explore the parameter space determined by triviality bounds and identify the regions where the $A^{0}$ can be detected at the LHC in each of the following modes: the inclusive two-photon decay mode, the $l^{ \pm} \gamma \gamma X$ mode from $t \bar{t} A^{0}$ production and the $A^{0} \rightarrow Z h$ channel with the subsequent decay of the $C P$-even scalar $h$ to two photons. We find that, while the $l^{ \pm} \gamma \gamma X$ mode is of limited usefulness, the other two modes can give viable signals in fairly large, and complementary, regions of parameter space.
\end{abstract}

*e-mail address: kominis@budoe.bu.edu 


\section{Introduction}

Despite the remarkable experimental success of the Standard Model of electroweak interactions, there is no evidence, so far, in favor of the minimal one-doublet scalar sector of the model. It is therefore essential that alternatives to it be considered. The simplest extension of the scalar sector of the Standard Model that naturally embodies certain indispensable features, such as the smallness of $\rho-1$ and the suppression of flavor-changing neutral currents, while admitting the possibility of new observable phenomena, is the (CP-conserving) two-Higgs-doublet model.

In the Standard one-doublet Model, the Higgs mass is constrained to lie between 57 and $800 \mathrm{GeV}$ approximately. The lower limit is set by direct searches at LEP [1], while the upper bound comes from theoretical considerations such as the triviality of theories with fundamental scalars [2, 3, 4]. The numerous phenomenological investigations of the one-doublet Standard Model [5] indicate that it may be possible to explore this entire range at future colliders such as LEP-II and the LHC. So we may ask about the potential of these colliders to detect alternative models. For a general two-Higgs-doublet model, a first step towards answering this question was made in [6], where the bounds on the parameters of the model that result from triviality considerations were derived. These bounds were obtained in the context of perturbation theory and so their validity is of a rather qualitative nature. To be definite, we will focus our interest on the region of parameter space defined by these triviality bounds. However, the general discussion we offer can give a fair idea of how to extend our results beyond this region.

In the analysis of ref. [6], following the spirit of [3], the term 'triviality' was employed in the following sense: The running scalar self-couplings of the Two-Doublet Model are expected, at least in perturbation theory, to develop a Landau pole at a finite momentum scale. Consequently, the theory can only stand as an effective low-energy theory valid up to some finite cutoff $\Lambda$, beyond which new phenomena emerge. The calculation of physical quantities in the effective theory will thus be accurate up to terms of order $p_{i}^{2} / \Lambda^{2}, M_{j}^{2} / \Lambda^{2}$, where $p_{i}$ are typical momenta of the process under consideration and $M_{j}$ are the masses of the particles of the theory. In ref. [6], the effective theory was defined to be valid if all masses satisfied

$$
\frac{M_{j}}{\Lambda} \leq \frac{1}{2 \pi}
$$

Thus, given a set of parameters of the model, that is, masses and couplings, a cutoff was defined by

$$
\Lambda=2 \pi \max _{j} M_{j}
$$

and the following consistency requirements were made: (i) No coupling should develop a 
Landau pole at a scale less than $\Lambda$, and (ii) the effective potential should be stable for all field values less than $\Lambda$. From these two conditions follow the 'triviality bounds' reported in [6] and used in the present study.

It is thus our intention to find out how much of the parameter space allowed by triviality can be explored at present and future colliders. In this paper we concentrate on the phenomenology of the $C P$-odd scalar (which we denote $A^{0}$ ) of the Two-Doublet Model at the LHC, and identify those regions of parameter space where direct observation of this particle is possible.

We examine three of the most promising signals for the $A^{0}$. First, we discuss the inclusive two-photon channel. As is the case for the Standard Higgs, a light $A^{0}$ decays predominantly into $b \bar{b}$ pairs and thus its detection at a hadron collider has to rely on rare modes, such as the two-photon mode. This signal usually consists of a large number of events and, furthermore, it may be useful even beyond the so-called 'intermediate mass' range, because the $A^{0}$ does not couple to a pair of weak gauge bosons at tree level. On the other hand, it is well known that there are very large backgrounds to this signal, whose successful containment places severe requirements on the design of the detector 17. Consequently, even a large event rate may not constitute a clean signal. For this reason, we also examine the process in which the $A^{0}$ is produced in association with a $t \bar{t}$ pair and decays into two photons while one of the $t / \bar{t}$ is tagged by its leptonic decay [8.9. The lepton tag ensures a much cleaner signal, but the event rate is so small as to render this mode only marginally useful. Finally, we turn to the discussion of the decay $A^{0} \rightarrow Z h$, where $h$ is a $C P$-even scalar, with the subsequent decay of $h$ in two photons, and demonstrate that it gives a very clear signal in a region of parameter space where none of the two other modes considered can be useful. To our best knowledge, this process has not been considered before in this context. Our results indicate that it may provide an excellent way of directly observing the CP-odd scalar $A^{0}$.

Phenomenological studies of two-Higgs-doublet models have been carried out mostly in the context of the Minimal Supersymmetric Model (MSSM) 10, 11, 12, 13, 14, 15,; if the supersymmetric partners are heavy enough, then the low-energy spectrum and the formal structure of the couplings of this model are exactly the same as in the more general non-supersymmetric case. Supersymmetry, however, imposes relations among the various masses and couplings, which reduce the number of independent parameters and allow for more definite predictions. Moreover, the parameter space usually investigated in studies of the MSSM is motivated by supersymmetric unification, while in the present study it is defined by the above-mentioned triviality bounds and, as a result, it is significantly different. It might be thought that the MSSM should really be contained in the more general two-doublet model as a special case. The models studied here, however, employ an exact discrete symmetry (see Section 2 and ref. [16]) intended to suppress flavor-changing 
neutral currents at tree level; if this symmetry is allowed to be softly broken, as is the case in the MSSM, most triviality bounds no longer hold [6]. In this case the parameter space of the MSSM is indeed a subset of that of the more general two-doublet model.

The larger number and different range of the parameters of the Two-Doublet Model may result in a markedly different phenomenology from that of the MSSM. A striking example is the $A^{0} \rightarrow Z h \rightarrow Z \gamma \gamma$ decay sequence, described in detail in Section 7 . We will further comment on the similarities and differences between the supersymmetric and non-supersymmetric two-doublet models in the course of the discussion of our results.

In the next section we review the Two-Doublet Model, emphasizing the features most relevant to phenomenology. In Section 3 we describe the production mechanisms of the $C P$-odd Higgs $A^{0}$ at the LHC. In Section 4 we discuss the main decay channels and present branching ratios of the $A^{0}$ in order to motivate our choice of the particular signals we subsequently examine. Section 5 concerns the inclusive two-photon channel, while in Section 6 we study the $l \gamma \gamma X$ signal from $t \bar{t} A^{0}$ production. Section 7 is devoted to the discussion of the decay $A^{0} \rightarrow Z h$. The results we present in Sections 5-7 consist in event-rate and significance contours in appropriate sections of parameter space, for integrated luminosities of $10 \mathrm{fb}^{-1}$ and $100 \mathrm{fb}^{-1}$, which correspond to a low- and a highluminosity option at the LHC. We have used a center-of-mass energy of $\sqrt{s}=16 \mathrm{TeV}$ in all calculations. Finally, Section 8 contains our conclusions.

\section{The two-doublet model}

The scalar sector contains two electroweak doublets $\Phi_{1}, \Phi_{2}$, both of hypercharge $Y=1$. In order to eliminate flavor-changing neutral currents at tree level, one has to impose a discrete symmetry [16]. There are many ways of doing this, the two most often discussed being the following [17,18:

- Model I : $\Phi_{1} \rightarrow-\Phi_{1}$

- Model II $\quad \Phi_{1} \rightarrow-\Phi_{1} \quad ; \quad d_{R i} \rightarrow-d_{R i}, \quad e_{R i} \rightarrow-e_{R i}$

$\left(d_{R i}(i=1,2,3)\right.$ are the right-handed negatively charged quarks and $e_{R i}$ the right-handed charged leptons.) The Lagrangian is

$$
\mathcal{L}=\mathcal{L}_{k i n}+\mathcal{L}_{Y}-V
$$

\footnotetext{
${ }^{1}$ The notation here has been changed from that of ref. [6] to facilitate comparison with most studies of the MSSM. Contact with [6] can be made by the following substitutions: $\Phi_{1} \leftrightarrow \Phi_{2}$, Model I $\leftrightarrow$ Model II. In terms of the angles $\alpha$ and $\beta$ introduced later in this Section, this has the effect $\alpha \leftrightarrow-\alpha, \beta \leftrightarrow \pi / 2-\beta$.
} 
where $\mathcal{L}_{\text {kin }}$ contains all the covariant derivative terms, $V$ is the scalar potential and $\mathcal{L}_{Y}$ contains the fermion-scalar interactions. The form of the latter is the following:

- Model I

$$
\mathcal{L}_{Y}=g_{i j}^{(u)} \bar{q}_{L i} \Phi_{2}^{c} u_{R j}+g_{i j}^{(d)} \bar{q}_{L i} \Phi_{2} d_{R j}+g_{i j}^{(l)} \bar{l}_{L i} \Phi_{2} e_{R j}+\text { h.c. }
$$

- Model II

$$
\mathcal{L}_{Y}=g_{i j}^{(u)} \bar{q}_{L i} \Phi_{2}^{c} u_{R j}+g_{i j}^{(d)} \bar{q}_{L i} \Phi_{1} d_{R j}+g_{i j}^{(l)} \bar{l}_{L i} \Phi_{1} e_{R j}+\text { h.c. }
$$

where $q_{L i}$ are the left-handed quark doublets, $u_{R i}$ are the up-type right-handed quarks and $l_{L i}$ are the left-handed lepton doublets. The matrix $g^{(l)}$ is understood to be diagonal.

Thus in Model I only $\Phi_{2}$ couples to fermions, while in Model II $\Phi_{1}$ gives mass to down-type quarks and leptons and $\Phi_{2}$ to up-type quarks. Because of the different fermion couplings, the two models have different phenomenology. We will be presenting results for each of the two models'?

The discrete symmetry (3) also limits the number of scalar self-couplings. The scalar potential is

$$
\begin{aligned}
V= & \mu_{1}^{2} \Phi_{1}^{\dagger} \Phi_{1}+\mu_{2}^{2} \Phi_{2}^{\dagger} \Phi_{2}+\lambda_{1}\left(\Phi_{1}^{\dagger} \Phi_{1}\right)^{2}+\lambda_{2}\left(\Phi_{2}^{\dagger} \Phi_{2}\right)^{2}+\lambda_{3}\left(\Phi_{1}^{\dagger} \Phi_{1}\right)\left(\Phi_{2}^{\dagger} \Phi_{2}\right) \\
& +\lambda_{4}\left(\Phi_{1}^{\dagger} \Phi_{2}\right)\left(\Phi_{2}^{\dagger} \Phi_{1}\right)+\frac{1}{2} \lambda_{5}\left[\left(\Phi_{1}^{\dagger} \Phi_{2}\right)^{2}+\left(\Phi_{2}^{\dagger} \Phi_{1}\right)^{2}\right]
\end{aligned}
$$

Provided certain inequalities among the scalar self-couplings are satisfied [19, the doublets $\Phi_{1}, \Phi_{2}$ acquire vacuum expectation values of the following form:

$$
\left\langle\Phi_{1}\right\rangle=\frac{1}{\sqrt{2}}\left(\begin{array}{c}
0 \\
v_{1}
\end{array}\right) \quad\left\langle\Phi_{2}\right\rangle=\frac{1}{\sqrt{2}}\left(\begin{array}{c}
0 \\
v_{2}
\end{array}\right)
$$

where $v_{1}, v_{2}$ are real and

$$
v_{1}^{2}+v_{2}^{2} \equiv v^{2}=(246 \mathrm{GeV})^{2}
$$

Note that $C P$ is a good symmetry of the scalar sector and so we may assign definite $C P$ quantum numbers to all neutral scalar states. At higher loop level, interactions with the fermions will invalidate this statement and mixings between $C P$-odd and $C P$-even states will appear due to the $C P$-violating phases of the Kobayashi-Maskawa matrix. These mixings, however, are small, and we will therefore neglect them in what follows.

\footnotetext{
${ }^{2}$ It should be noted that the MSSM is of type II only.
} 


\begin{tabular}{|c|c|c|}
\hline & Model I & Model II \\
\hline$h V V$ & $\sin (\beta-\alpha)$ & $\sin (\beta-\alpha)$ \\
\hline$H V V$ & $\cos (\beta-\alpha)$ & $\cos (\beta-\alpha)$ \\
\hline$A^{0} V V$ & 0 & 0 \\
\hline$h u \bar{u}$ & $\cos \alpha / \sin \beta$ & $\cos \alpha / \sin \beta$ \\
\hline$h d \bar{d}$ & $\cos \alpha / \sin \beta$ & $-\sin \alpha / \cos \beta$ \\
\hline$H u \bar{u}$ & $\sin \alpha / \sin \beta$ & $\sin \alpha / \sin \beta$ \\
\hline$H d \bar{d}$ & $\sin \alpha / \sin \beta$ & $\cos \alpha / \cos \beta$ \\
\hline$A^{0} u \bar{u}$ & $i \gamma^{5} \cot \beta$ & $i \gamma^{5} \cot \beta$ \\
\hline$A^{0} d \bar{d}$ & $-i \gamma^{5} \cot \beta$ & $i \gamma^{5} \tan \beta$ \\
\hline
\end{tabular}

Table 1: Weak gauge boson and fermion couplings of the neutral scalars of the two-doublet model relative to the corresponding Standard Higgs couplings.

In addition to the Goldstone bosons which become the longitudinal components of the $W$ 's and the $Z$ via the Higgs mechanism, the spectrum of the scalar sector contains two neutral $C P$-even scalars, denoted by $h, H$, one neutral $C P$-odd scalar, $A^{0}$, sometimes referred to as a pseudoscalar due to its $\gamma^{5}$ coupling to fermions, and two charged complex conjugate states $H^{ \pm}$. It is customary to introduce two mixing angles: $\beta\left(=\arctan \left(v_{2} / v_{1}\right)\right)$ rotates the $C P$-odd and the charged scalars into their mass eigenstates while $\alpha(-\pi / 2<\alpha \leq \pi / 2)$ rotates the neutral scalars into their mass eigenstates. There are six independent parameters in the scalar potential (after fixing $v$ through (8)) which can be taken to be $\alpha, \beta, M_{H^{ \pm}}, M_{H}, M_{h}, M_{A^{0}}$. (In contrast, in the MSSM there are only two.) To these one should add the top quark mass, expected to lie in the range [20]

$$
100 \mathrm{GeV} \lesssim M_{t} \lesssim 200 \mathrm{GeV}
$$

but otherwise unspecified.

In Table 1 we display the couplings of the neutral scalars to vector bosons and fermions for Models I and II. The values shown are relative to the Standard Model Higgs couplings; $V$ denotes collectively the weak gauge bosons, $u$ the up-type quarks and $d$ the down-type quarks and leptons. We also display below the couplings of $A^{0}$ to a scalar and a vector boson, since they are important in the determination of the partial widths of $A^{0}$.

$\mathcal{L}_{A^{0} S V}=\frac{1}{2} \sqrt{g^{2}+g^{\prime 2}} Z_{\mu}\left[A^{0} \stackrel{\leftrightarrow}{\partial^{\mu}}(H \sin (\beta-\alpha)-h \cos (\beta-\alpha))\right]-\frac{1}{2} g W_{\mu}^{+}\left(A^{0} \stackrel{\leftrightarrow}{\partial^{\mu}} H^{-}\right)+$h.c.

where $g^{\prime}, g$ are the $U(1)_{Y}$ and $S U(2)$ gauge couplings respectively. The full set of Feynman rules can be found in ref. [17]. 
Of crucial importance to the phenomenology of the $C P$-odd Higgs $A^{0}$ is the absence of a tree level coupling between it and a pair of weak gauge bosons. This deprives us of a very clean signal as well as of an important production mechanism for masses of a few hundred GeV. It is also evident from Table 1 that the couplings of the Higgs bosons to fermionantifermion pairs can be significantly enhanced or suppressed over those of the Standard Higgs depending on the values of $\beta$ and $\alpha$ and the choice of Model. Similar suppressions can occur in the couplings of the $A^{0}$ to the other neutral scalars (see eq. (10)). Unlike the situation in the MSSM, these couplings are independent of the scalar masses. This freedom has important consequences for phenomenology, as will be demonstrated in later sections.

The strongest experimental constraints on the two-doublet model arise from the recent CLEO bound on $B R(b \rightarrow s \gamma)$ [21] and from LEP data on the $Z \rightarrow b \bar{b}$ decay width 222. These measurements impose a combined bound on $\beta$ and $M_{H^{ \pm}}$[23,24. There are uncertainties [23 about the precise value of these bounds, and we will not include them in our analysis. From refs. [23,24, however, it is clear that light charged scalars $\left(M_{H^{ \pm}} \lesssim 50 \mathrm{GeV}\right)$ and low $\beta\left(\beta \lesssim 15-20^{\circ}\right)$ are strongly disfavoured, with the restrictions becoming tighter for larger top quark masses.

\section{Production mechanisms}

As was emphasized earlier, the coupling of the $A^{0}$ to intermediate vector bosons is induced only at loop level and is therefore small. So, in contrast to the case of the Standard Higgs, production of the $A^{0}$ via vector boson fusion or in association with a $W$ or a $Z$ is insignificant. Thus, at a hadron collider, we have to rely on the following mechanisms:
(a) Gluon fusion
(b) Associated $b \bar{b} A^{0}$ production
$g g \rightarrow A^{0}$
(c) Associated $t \bar{t} A^{0}$ production
$q \bar{q}, g g \rightarrow b \bar{b} A^{0}$
$q \bar{q}, g g \rightarrow t \bar{t} A^{0}$

The cross sections for processes (a)-(c) are identical to the corresponding rates in the MSSM, since they only depend on $M_{A^{0}}, M_{t}$ and $\tan \beta$. We discuss them here for completeness and for future reference. Our results are in agreement with ref. [13.

\subsection{Gluon fusion}

This is usually the dominant mode. The reaction proceeds via a quark loop [25]. The contribution of the top quark is generally the most significant, but, in Model II and for 
large $\beta$, the bottom quark contribution can become equally important or even dominate, especially for small $M_{A^{0}}$. We have taken into account leading QCD corrections as follows: in ref. [26] the QCD corrections to the production of a pseudoscalar have been calculated in the limit of a very heavy quark flowing in the loop. The major part of these corrections can be cast into the form

$$
\sigma=\sigma_{0}\left[1+\left(6+\pi^{2}\right) \frac{\alpha_{s}\left(M_{A^{0}}\right)}{\pi}\right]
$$

in analogy to the case of a $C P$-even Higgs [10]. $\sigma_{0}$ and $\sigma$ are the lowest-order and QCDcorrected cross-sections respectively. For our purpose, this is a valid approximation only if $M_{A^{0}}<2 M_{t}$ and only insofar as the bottom loop contribution, to which this correction does not apply, is negligible compared to that of the top quark. With hindsight, we may say that the second qualification is not worrisome: in the regions where the bottom quark contribution is significant, namely large $\beta$, small $M_{A^{0}}$, the rates for the signals we will be interested in are small anyway. This may not be so for $\beta$ very close to $90^{\circ}$, but triviality bounds almost always exclude such values of $\beta$. The QCD corrections to arbitrary mass pseudoscalar production from gluon fusion have not been computed. Consequently, we shall only employ (11) if $M_{A^{0}}<2 M_{t}$.

Figures 1a, 1b show the production cross-section of the $C P$-odd Higgs through gluon fusion as a function of its mass for Models I and II respectively. The effect of the bottom quark loop for large $\beta$ in Model II is evident. The top quark mass was taken to be $150 \mathrm{GeV}$. The curves for different $M_{t}$ are similar; they always peak at $M_{A^{0}}=2 M_{t}$, as

long as $\beta$ does not take on values extremely close to $90^{\circ}$ in Model II. The value at the peak is lower for higher $M_{t}$. Here and throughout this study we employed the EHLQ structure functions, Set 2 , with $\Lambda_{\overline{M S}}=290 \mathrm{MeV}$ [27.

\subsection{Associated $b \bar{b} A^{0}$ production}

This mechanism can be important for large $\beta$ in Model II when the $b \bar{b} A^{0}$ coupling becomes strong. For $M_{A^{0}} \gg M_{b}$, this process can be approximated [28] by $b \bar{b}$ fusion using the bottom quark distribution functions within the proton. The $b \bar{b}$ fusion cross-section is proportional to the partial width $\Gamma\left(A^{0} \rightarrow b \bar{b}\right)$. QCD corrections to the latter have been included in our calculation as we explain in Section 3. No other QCD corrections to this process have been estimated.

In Figures 2a, 2b we show the production cross-section of $A^{0}$ from $b \bar{b}$ fusion as a function of the $A^{0}$ mass for Models I and II. It can be seen that, in the case of Model I, this cross-section never exceeds the gluon fusion cross-section. In contrast, this mechanism dominates in Model II for very large values of $\beta$. 


\subsection{Associated $t \bar{t} A^{0}$ production}

This process always gives a smaller rate than gluon fusion. It is interesting, however, in its own right, because it is possible to obtain a relatively clear signal if one can tag on a lepton from the decay of the top or antitop. This will be further discussed in Section 6 . We have computed this cross-section using the spinor techniques of ref. [29]. QCD corrections have not appeared in the literature and are not included. In Figures 3a, 3b we display the cross-section for the process $p p \rightarrow t \bar{t} A^{0}$ as a function of the pseudoscalar mass and for the set of values of $\beta$ used in the previous graphs. The top quark mass is $120 \mathrm{GeV}$ and $180 \mathrm{GeV}$ respectively. There is only a slight drop in rate with increasing top quark mass, as the smaller parton luminosities at high mass are compensated by the enhanced Yukawa coupling. There is no distinction between Models I and II in this case because the $A^{0} t \bar{t}$ coupling is the same in both Models (see Table 1). We also show the corresponding curves for the Standard Model Higgs. In all calculations we have included the contributions of both the $g g$ and $q \bar{q}$ initial states. The latter is negligible for the $C P$ odd scalar but amounts to 20-25\% of the gluon-initiated reaction for a Standard Higgs lighter than about $200 \mathrm{GeV}$ (see also [30]). We notice that the curves for the $C P$-odd Higgs are much flatter than those for the Standard Higgs. For $\beta=45^{\circ}$, the $t \bar{t} A^{0}$ production rate for $A^{0}$ masses less than about $150 \mathrm{GeV}$ is significantly lower than the corresponding rate for the Standard Higgs. As we will see in Section 6, this has unpleasant phenomenological implications.

In Figures $4 \mathrm{a}, 4 \mathrm{~b}$ we show the total production cross-section for the $A^{0}$, the sum, that is, of the three processes discussed above. Results are shown for the two Models and for the same values of $\beta$ used in previous figures. A top quark mass of $150 \mathrm{GeV}$ was assumed. We again display the corresponding cross-section for the Standard Higgs. (Gauge boson fusion has not been included in the calculation of the latter.) The conclusions to be drawn from these graphs are the following: (i) except for very large values of $\beta$ in Model II, the production cross-section increases with decreasing $\beta$; (ii) the two Models diverge only at large $\beta$; (iii) for $\beta \lesssim 50^{\circ}$ the cross-section is larger than that of the Standard Higgs; (iv) the cross-section is generally peaked at $M_{A^{0}}=2 M_{t}$. Variation of $M_{t}$ shifts the peak accordingly and lowers the value at the peak somewhat. The other parameters of the two-doublet model do not affect the production rate of the $C P$-odd scalar.

\section{Decays and Branching Ratios}

In this Section we review the major decay modes and the corresponding branching ratios of the $C P$-odd Higgs in order to identify the signals that can be of phenomenological interest. In the absence of a tree level coupling to the $W$ 's and the $Z$, the $A^{0}$ decays 
mainly to fermion pairs or to states containing other scalars. Loop-induced decays can also be large, like the two-gluon mode, or phenomenologically interesting, like the twophoton decayf. The main channels are the following:

$$
A^{0} \rightarrow b \bar{b}, c \bar{c}, t \bar{t}, \tau^{+} \tau^{-}, W^{ \pm} H^{\mp}, Z h, Z H, g g, \gamma \gamma, Z \gamma
$$

All decay widths have been calculated to lowest non-trivial order. Analytic formulae for the last three, loop-induced, decays can be found in the literature [31, 17, 13]. QCD corrections have been included where they are most significant, namely:

(i) the $q \bar{q}$ decays $(q=b, c)$ : Leading QCD effects have been accounted for by using, in the expression for the Yukawa coupling, the running quark mass at the scale of the mass of the $C P$-odd Higgs [32;

(ii) the $\gamma \gamma$ and $Z \gamma$ decays through quark loops: Corrections have been calculated in ref. 33 for the $\gamma \gamma$ decay. The leading contribution can be incorporated by using the running quark mass at scale $M_{A^{0}}$. The remaining QCD corrections are mostly within $10 \%$ and have not been included. We have also used running masses in the computation of the decay to $Z \gamma$;

(iii) the $g g$ decay: Corrections to this decay have only been computed for the case of a $C P$-even Higgs [34]. In analogy to that calculation, we used running quark masses in the loops, but did not include any further corrections.

The branching ratios of the $C P$-odd scalar are complicated functions of the seven free parameters of the model and hence impossible to describe completely. We will illustrate some of the most prominent features by means of a few examples.

Fig. 5 shows the branching ratios of the $A^{0}$ as a function of its mass for a case where the other scalars, $h, H$ and $H^{ \pm}$, are heavier than the top quark. The set of values of the parameters chosen (referred to as Set A) is the following:

$$
\begin{array}{ll}
\text { Set } \mathrm{A}: \quad M_{H}=400 \mathrm{GeV}, & M_{h}=260 \mathrm{GeV}, \quad M_{H^{ \pm}}=350 \mathrm{GeV}, \\
& M_{t}=120 \mathrm{GeV}, \quad \alpha=30^{\circ} .
\end{array}
$$

Branching ratios are shown for two values of $\beta\left(30^{\circ}\right.$ and $\left.60^{\circ}\right)$ and for Models I and II. Of the decay channels listed in (12) we do not show here the branching ratios to $c \bar{c}, g g, Z H$ and $Z \gamma$. The first two are phenomenologically uninteresting at hadron colliders. The $Z H$ channel has a behaviour qualitatively similar to that of the $Z h$ mode, while the branching ratio to $Z \gamma$, a channel of potential phenomenological importance, is generally very small. We point out the following features: (i) For $M_{A^{0}}<2 M_{t}$, the dominant decay mode is $b \bar{b}$,

\footnotetext{
${ }^{3}$ See [13], however, for an investigation of the mode $A^{0} \rightarrow Z Z$ in the MSSM.
} 
unsuitable for detection of a Higgs at a hadron collider. Thus, the $\gamma \gamma$ or $\tau^{+} \tau^{-}$decays have to be employed. (ii) Whereas the branching ratio to two photons in Model I is insensitive to $\beta$, in Model II it is a decreasing function of $\beta$, at least for moderate $\beta$ values. This is easy to understand; the two-photon width is governed by the $A^{0} t \bar{t}$ coupling, because of the top loop that mediates the decay, and so goes like $\cot ^{2} \beta$ (see Table 1), whilst the full width is essentially saturated by the $b \bar{b}$ mode $\left(\sim \tan ^{2} \beta\right)$. As a result, the $\gamma \gamma$ branching ratio has an approximate $\cot ^{4} \beta$ dependence. In Model I, however, the $A^{0}$ couplings to $b \bar{b}$ and $t \bar{t}$ are both proportional to $\cot \beta$ and the $\beta$-dependence drops out. (iii) The channels that contain scalars $\left(Z h, W^{ \pm} H^{\mp}\right)$ become dominant, or at least compete with the $t \bar{t}$ mode, as soon as they open. (iv) Both the $\tau^{+} \tau^{-}$and the $\gamma \gamma$ branching ratios fall abruptly once the $t \bar{t}$ channel becomes available. In the region between the $t \bar{t}$ and the scalar particle thresholds, detection of the $A^{0}$ seems dubious.

With Fig. 6 we wish to illustrate the dependence of the branching ratios on the angle $\alpha$ instead. In what we call Set B in this figure, the parameters have the following values:

$$
\begin{array}{ll}
\text { Set B : } \quad M_{H}=400 \mathrm{GeV}, \quad M_{h}=100 \mathrm{GeV}, \quad M_{H^{ \pm}}=350 \mathrm{GeV}, \\
M_{t}=180 \mathrm{GeV}, \quad \alpha=30^{\circ},
\end{array}
$$

whereas in 'Set C' they are

$$
\begin{array}{ll}
\text { Set } \mathrm{C}: & M_{H}=400 \mathrm{GeV}, \quad M_{h}=100 \mathrm{GeV}, \quad M_{H^{ \pm}}=350 \mathrm{GeV}, \\
& M_{t}=180 \mathrm{GeV}, \quad \alpha=-45^{\circ} .
\end{array}
$$

$\beta$ is fixed at $40^{\circ}$ in all graphs of this figure. This is an example of the $Z h$ channel opening up before the $t \bar{t}$ does. The main points we want to emphasize here are the following: (i) $\gamma \gamma$ and $\tau^{+} \tau^{-}$are again the only possibilities for detection of a light $A^{0}$; (ii) the $\alpha$-dependence comes from the $A^{0} Z h$ coupling (see eq. (10)) which is proportional to $\cos (\beta-\alpha)$. In Set $\mathrm{B}$ this coupling is not suppressed and the $Z h$ mode is dominant even after the $t \bar{t}$ channel becomes available. In contrast, in Set $\mathrm{C}$, the $Z h$ branching ratio is sizeable only before the $t \bar{t}$ threshold. What is also to be noticed in this case is that the opening of the $Z h$ channel does not cause any significant drop to the value of the two-photon branching ratio. As a result, the $\gamma \gamma$ decay remains a promising mode for $A^{0}$ masses as large as $2 M_{t}$. It should be noted that no such suppression is present in the $A^{0} W^{ \pm} H^{\mp}$ coupling (see eq. (10)). Consequently, the two-photon branching ratio necessarily drops once the $W^{ \pm} H^{\mp}$ channel is open, independently of the value of $\alpha$.

Although the particular shapes of these curves change for different values of the TwoDoublet Model parameters, the general features described above persist. We are thus led

\footnotetext{
${ }^{4} \beta=30^{\circ}$ in Model II illustrates the case of the $g g$ branching ratio (not shown) becoming dominant just below the top threshold.
} 
to examine the observability of the $C P$-odd Higgs in the two-photon mode (as the most promising alternative for a light $A^{0}$, but potentially useful even for an $A^{0}$ of mass as large as $2 M_{t}$ ) and in the $Z h$ mode (which, unless it is suppressed, covers a range of higher masses $\left.M_{A^{0}}\right)$. We have not further considered the $\tau^{+} \tau^{-}$mode .

\section{The inclusive two-photon channel}

This mode has been extensively studied in the literature for the $C P$-odd scalar of the MSSM [10,12, 14, 15]. Since the parameter space under investigation here is qualitatively rather different, as explained in the Introduction, an independent study is necessary. We shall briefly compare our findings to those for the MSSM, to the extent that this is possible, at the end of this Section.

To assess the observability of a Higgs signal in this mode a reliable calculation of the reducible and irreducible backgrounds is required. This, in turn, presupposes a good understanding of the detector parameters and a full Monte Carlo simulation. We did not make such an effort here, but only tried to approximate a realistic situation as much as possible, within the framework of simple parton-level calculations. Our procedure of estimating the backgrounds, which are the same as in the Standard Model, is sketched below.

At lowest order, the direct two-photon background arises from the Born process

$$
q \bar{q} \rightarrow \gamma \gamma
$$

The gluon fusion process

$$
g g \rightarrow \gamma \gamma
$$

through the 'box' diagram, gives a contribution of the same order of magnitude as (16). We calculated the cross-sections for processes (16) and (17) using the following kinematical cuts to simulate the detector acceptance:

$$
\left|\eta^{\gamma}\right|<2.5 \quad ; \quad p_{T}^{\gamma}>20 \mathrm{GeV}
$$

In the calculation of the 'box' diagram we used five light flavours and a top quark of $150 \mathrm{GeV}$. Variation of the top quark mass in the range $100 \mathrm{GeV}<M_{t}<200 \mathrm{GeV}$ produces

\footnotetext{
${ }^{5}$ See [10] for a discussion of this channel in the MSSM. The $\tau^{+} \tau^{-}$branching ratio for a light $A^{0}$ is of the order of $10 \%$ in both the MSSM and the Two-Doublet Model; consequently, the findings of [10] should apply here too.
} 
negligible change in the cross-section. A next-to-leading order calculation of the di-photon background [35.36] shows that bremsstrahlung processes, where one or more photons are radiated off a jet, largely dominate over the lowest order ones. However, suitable isolation cuts [36] can reduce this higher-order background to about half the sum of the Born and 'box' contributions. We therefore multiplied the calculated cross-section of processes (16) and (17) by a 'K-factor' of 1.5 in order to 'convert' to the next-to-leading-order result.

The background from events where jets are faking photons can be significant. We assumed (following [37, 38]) that it will be possible to achieve such a jet rejection efficiency as to reduce these backgrounds to well below the prompt di-photon level. In fact, even so, the jet backgrounds can still be as large as $25 \%$ of the physical $\gamma \gamma$ background [37, depending on the two-photon invariant mass. We adopted this figure as our estimate of the jet background that remains after all isolation cuts, applying it to all invariant masses. Finally, we assumed a 90\% reconstruction efficiency for each photon as well as a further $7 \%$ loss, from isolation cuts, due to pileup, if the high luminosity option is used [37. To obtain the number of background events, we used a mass bin of $\Delta M_{\gamma \gamma}=3 \% M_{\gamma \gamma}$. This is probably a conservative choice, given the energy resolution capability quoted in refs. [37.38]. However, precise vertex identification is a very difficult task [7], especially at high luminosity, and it is uncertain how accurately the vertex will be localized [37]. A conservative assumption on the vertex identification capability leads to an energy smearing such that our choice of $\Delta M_{\gamma \gamma}$ then corresponds, approximately, to a $2 \sigma$ bin width (see [37). In the cases where the signal was broader than this mass bin, we summed the background over the width of the resonance.

The signal event rate depends on all six parameters of the scalar sector as well as on the top quark mass and the choice of Model. The discussion of Sections 3 and 4 has shown that the most important parameter, besides $M_{A^{0}}$, is $\beta$, by virtue of its effect on both the production rate and the two-photon decay width of the $C P$-odd Higgs. We will therefore present results fixing the other parameters at first. Nonetheless, we will discuss the effect of varying them and illustrate it by means of some examples.

In Section 4 it was observed that the widths of the $t \bar{t}$ and scalar decay modes are generally large, so that the branching ratio to two photons is, most of the times, greatly reduced once these channels are open. Figures 7 and 8 reflect this behaviour. They display the production cross-section times the two-photon branching ratio of the $A^{0}$ as a function of its mass for various values of $\beta$. Fig. 7 corresponds to Set A of the parameters (see (13)), while Fig. 8 corresponds to Set B (eq. (14)). We also plot, in each case, the same quantity for the Standard Higgs. In these calculations we included QCD corrections, as explained in Sections 3 and 4, but not cuts or efficiencies. The curves do not therefore represent the actual signal cross-sections, but, rather, they are shown for purposes of comparison. As expected from the discussion of the preceding sections, the rate increases with $M_{A^{0}}$ until 
the $t \bar{t}$ or $Z h$ threshold is met; beyond this, the rate falls due to the sharp decrease of the $\gamma \gamma$ branching ratio. In Fig. 8, the cross-section rises again right before $M_{A^{0}}=2 M_{t}$, reflecting the increased production rate at this mass. (The only exception to this general pattern is the case of large $\beta$ in Model II; there, the quark loop diagrams responsible for both the $A^{0}$ production from gluon fusion and the decay to two photons are dominated by the bottom, rather than the top, quark and so do not possess a maximum at $M_{A^{0}}=2 M_{t}$.) Variation of $M_{t}, M_{H^{ \pm}}, M_{H}, M_{h}$ may change the order in which the various thresholds appear, but has little effect otherwise. The dependence on the angle $\alpha$ is generally mild, except for its influence on the couplings of $A^{0}$ to $Z h$ and $Z H$, discussed at length in Section 4 . Figure 9 corresponds to Set $\mathrm{C}$ of parameters (see (15)), an example where the suppression of the $A^{0} Z h$ coupling is particularly strong.

Comparison with the Standard Higgs, in Figures 7-9, shows that in the so-called intermediate-mass range, the rates for the $A^{0}$ are smaller, unless $\beta \lesssim 40^{\circ}$. On the other hand, they can remain substantial for a much wider range, reflecting the fact that the $W W$ and $Z Z$ decay modes are effectively absent for the $C P$-odd scalar.

In order to determine the statistical significance of a signal in this mode, we computed its rate using the cuts (18) and the efficiencies quoted in the discussion of the backgrounds. We only accepted signals that consist of at least 40 events and defined the significance by the ratio $S / \sqrt{B}$. In Figs. 10-12, we plot $5 \sigma$ contours for integrated luminosities of $10 \mathrm{fb}^{-1}$ and $100 \mathrm{fb}^{-1}$, and for each of Sets A, B and C. The contour corresponding to $10 \mathrm{fb}^{-1}$ can also be regarded as a $15 \sigma$ contour 0 for an integrated luminosity of $100 \mathrm{fb}^{-1}$; it thus serves also as an indication of the variation of the significance in the parameter space depicted. As explained in the Introduction, we restrict attention to the region of parameter space allowed by triviality constraints; this region is shown explicitly in Figs. 10-12. The significance plots show somewhat more quantitatively the behaviour expected on the basis of the results displayed in Figs. 7-9: For Sets A and B the opening of the $t \bar{t}$ and $Z h$ channels respectively, makes the detection of $A^{0}$ virtually impossible beyond these thresholds, despite the small window around $M_{A^{0}}=2 M_{t}$ (Set B). On the other hand, the suppression of the strength of the $A^{0} Z h$ coupling leads to rather spectacular results for Set C. It is worth noting that this is not a case of fine-tuning: any $\alpha$ in the range $-60^{\circ} \lesssim \alpha \lesssim-30^{\circ}$ would have more or less a similar effect.

Our comment on the dependence of the curves of Figs. 7-9 on the values of the other parameters of the model can help one visualize the variation of these significance contours with $M_{t}, M_{H^{ \pm}}, M_{H}, M_{h}$ and $\alpha$ : we expect the masses to play a role only insofar as they

\footnotetext{
${ }^{6}$ Here and in subsequent Sections we effectively scale the number of signal and background events with the integrated luminosity (except for the small pileup effect mentioned earlier). It is not absolutely clear, however, whether the assumed detector efficiency can be maintained in a high luminosity environment [7.
} 
set the various thresholds, and $\alpha$ to smoothly effect the transition from a situation like that of Fig. 11 to one like that of Fig. 12, as it gradually alters the factor of $\cos (\beta-\alpha)$ governing the $Z h$ branching ratio of the $A^{0}$. (The latter is relevant only if the $Z h$ threshold comes before the $t \bar{t}$ or $W^{ \pm} H^{\mp}$ ones.) However, there is a different way in which all these parameters may affect our conclusions, and this is by changing the triviality bounds. The allowed area may be shifted to lower or higher $\beta$, expanded or shrunk according to the precise values of the parameters chosen. This effect won't be too drastic if the scalars and top quark are not very light or very heavy; however, an example is shown in Fig. 13, where the region in which the $A^{0}$ can be observed in this mode is rather narrow. We note the values chosen for this example for future reference:

$$
\begin{array}{ll}
\text { Set D : } \quad M_{H}=400 \mathrm{GeV}, \quad M_{h}=100 \mathrm{GeV}, \quad M_{H^{ \pm}}=350 \mathrm{GeV} \\
M_{t}=180 \mathrm{GeV}, \quad \alpha=-75^{\circ} .
\end{array}
$$

In the MSSM, values of $\tan \beta>1$ are preferred [14]. In this case, since $\alpha$ is not an independent parameter, $\cos (\beta-\alpha)$ is constrained to be small, especially for large $M_{A^{0}}$; thus the $A^{0} Z h$ coupling is suppressed, leading to a situation similar to that of Fig. $12 \mathrm{~b}$. However, the bias on $\tan \beta$ restricts us to a region of low production cross-section making this mode appear less valuable overall than in the Two-Doublet Model.

As a conclusion, we may say that there is a substantial region of parameter space where the inclusive two-photon mode provides a clear signal for the $A^{0}$. This region extends from the lowest allowed $\beta$ to $\beta \sim 55-60^{\circ}$, with the signal fading as $\beta$ increases, and from $M_{A^{0}} \sim 40 \mathrm{GeV}$ to the first threshold of a 'strong' decay (by which we mean $t \bar{t}, W^{ \pm} H^{\mp}$ or $Z h$ if not suppressed), with the signal improving with larger $M_{A^{0}}$. This is true for both Model I and Model II. From the examples considered it can be seen that Model II is slightly less favourable to high $\beta$ values than Model I, but otherwise exhibits similar behaviour. These results, however, were based on rather optimistic assumptions about the ability of the detector to successfully reduce the jet backgrounds. This uncertainty has led many authors to seek alternative methods of detecting the scalar particles of the Standard Model and its extensions [8,9, 39]. We now turn to a method that has recently attracted a lot of attention.

\section{$6 \quad$ The $l \gamma \gamma X$ channel}

In the Standard Model, the Higgs boson can be produced in association with a $W$ or a $t \bar{t}$ pair through the following processes:

$$
\begin{aligned}
q \bar{q} & \rightarrow W^{\star} \rightarrow W H \\
g g, q \bar{q} & \rightarrow t \bar{t} H
\end{aligned}
$$


The leptonic decay of the $W$ or the $t / \bar{t}$ gives rise to a final state that consists of two isolated photons from the Higgs and an isolated lepton $(e$ or $\mu)$. Although the event rate is not high, the signal is rather clean, at least at low luminosity [8,9, 40, 41, 12].

For the CP-odd scalar of the Two-Doublet Model, (20) gives a negligible rate, as stated earlier. Consequently, we concentrate on (21). The final state from this process contains, besides the photons and the lepton, at least two jets. This can be used to remove most of the reducible backgrounds [40,42], as will be explained below.

The irreducible backgrounds arise from the processes

$$
\begin{aligned}
& g g \rightarrow t \bar{t} \gamma \gamma \\
& q \bar{q} \rightarrow t \bar{t} \gamma \gamma
\end{aligned}
$$

We did not perform an independent calculation of these backgrounds, but relied instead on the results of ref. [12 for (22) ษ. The cuts assumed by these authors were the following:

$$
\begin{array}{ll}
\left|\eta^{l, \gamma}\right|<2.5 & p_{T}^{l, \gamma}>20 \mathrm{GeV} \\
\Delta R\left(\gamma_{1}, \gamma_{2}\right)>0.4 \quad & \Delta R(l, \gamma)>0.4
\end{array}
$$

Insisting that at least two jets be detectable in the final state results in a loss of rate that varies from $25 \%$ for $M_{t}=100 \mathrm{GeV}$ to $4 \%$ for $M_{t}=160 \mathrm{GeV}$ [12]. (In [42] a jet was deemed detectable if it passed the same cuts as a photon or a lepton.) The background (23) was calculated in ref. [30] and was found to amount to $50 \%$ of (22) at $M_{\gamma \gamma}=100 \mathrm{GeV}$ climbing to $120 \%$ at $M_{\gamma \gamma}=180 \mathrm{GeV}$. We assumed these values in our estimate of the background.

A potentially serious background could arise from the process $g g \rightarrow b \bar{b} \gamma \gamma$, where a $B$ meson decays semileptonically. Since, however, the matrix element for this process is peaked when the $b$ quarks are emitted along the beam direction, the cuts imposed on jets reduce this background to negligible levels [42]. Other reducible backgrounds arise from events involving $t \bar{t}$ production in which one or two jets in the final state are misidentified as photons. In [12], the background from $t \bar{t} g$ production was calculated and found negligible assuming a $\gamma$-jet rejection factor of $5 \times 10^{-4}$. A rough estimate of $t \bar{t} \gamma$ production was also reported and the resulting background declared to be small compared to the $t \bar{t} \gamma \gamma$ background, though not negligible. Another possible source of background is $t \bar{t}$ production, where both photons are faked by jets from top decays. We assumed that these backgrounds can all be kept below the irreducible $t \bar{t} \gamma \gamma$ level. However, a detailed study is necessary in order to assess their exact magnitude at the LHC.

\footnotetext{
${ }^{7}$ These authors employed the HMRSB parton distribution functions 43 . We scaled their results by 1.5 to account for the fact that EHLQ usually yield higher rates for gluon-initiated processes $[9]$.
} 
To obtain the background event number we used the reconstruction efficiency for photons and leptons (65\% overall) quoted in [37] and assumed, as before, a 3\% resolution for the invariant mass of the photons. The background is a decreasing function of the top quark mass, due to the smaller parton luminosities at higher energies. Moreover, it depends indirectly on the mass of the charged Higgs: if the decay mode $t \rightarrow H^{+} b$ is kinematically allowed, then the leptonic $(e$ and $\mu$ ) branching ratio of the top (or antitop) is reduced from the Standard Model value of $2 / 9$ because $H^{+}$decays to electrons or muons are very rare. This remark applies, of course, also to the signal cross-section, to the discussion of which we now turn.

Fig. 14 shows the cross-section times branching ratio for the process $p p \rightarrow t \bar{t} A^{0} \rightarrow$ $l \gamma \gamma X$, as a function of $M_{A^{0}}$ for various values of $\beta$. The other parameters are chosen to belong to Set A (see (13)). The corresponding curve for the Standard Higgs is also shown. Cuts were not included in this calculation. The variation of the curves with the top quark mass is illustrated in Fig. 15, where $M_{t}=180 \mathrm{GeV}$ while the remaining Two-Doublet Model parameters are as in Set A. We shall refer to this choice as 'Set E'. We note that, due to the weak dependence of the production cross-section on the mass of the top, the rates are not too different for Sets $\mathrm{A}$ and $\mathrm{E}$ as long as $M_{A^{0}}<2 M_{t}$. The production cross-section does not depend on parameters other than $M_{A^{0}}, M_{t}$ and $\beta$, while the variation of the branching ratio with the other parameters has been discussed in Section 4. Consequently, we expect, as before, a sudden drop of the signal rate when the $t \bar{t}$ or scalar thresholds appear, unless again there is a suppression of the $A^{0} Z h$ coupling. What is noteworthy in Figs. 14-15 is that for Higgs masses of less than about $160 \mathrm{GeV}$ and for a large range of values of $\beta$ the rate is well below its Standard Model value, especially in Model I. Considering the fact that a Standard Higgs of this mass can only give a few tens of events [9,40,41, we recognize that the prospects of detecting $A^{0}$ in this mode are rather poor. In compensation, however, the mass range where this mode could be - even marginally - helpful may be much wider.

In Figs. 16-17 we display significance contours for Sets A and E. Here the calculation of the signal was carried out including the cuts (24), reconstruction efficiencies and losses from insisting that at least two jets be detected in the final state. The fraction of signal lost due to this last requirement is the same as for the background (see ref. [42]). The kinematical cuts result in a signal loss that varies from $75 \%$ at $M_{A^{0}}=30 \mathrm{GeV}$ to about $30 \%$ at $M_{A^{0}}=300 \mathrm{GeV}$ (with a very soft dependence on the top quark mass). We required that a signal candidate should consist of more than 10 events. For low numbers of events, the formula for the significance used in Section 5 is not very accurate because it is based on Gaussian statistics. Since the production of 'events' is a Poisson process, 
the probability that an apparent signal is in fact due to background fluctuations is [7]

$$
P=\sum_{n=S+B}^{\infty} P_{n}(B)
$$

where $S, B$ are the numbers of expected signal and background events respectively and $P_{n}(B)$ is the probability that $n$ background events occur, given by the Poisson distribution

$$
P_{n}(B)=B^{n} e^{-B} / n !
$$

(Since $S+B$ is not an integer in general, an interpolation between the two integers closest to it is employed.) The probability $P$ can then be converted into a significance.

The contour for an integrated luminosity of $10 \mathrm{fb}^{-1}$ is absent from Figs. 16-17; at such low luminosity the LHC cannot detect the $C P$-odd scalar of the two-doublet model in this mode at the $5 \sigma$ confidence level. For Set E, Model I, even a luminosity of $100 \mathrm{fb}^{-1}$ is inadequate. The main problem is not the background, which is small, especially for large $M_{t}$, but rather the size of the signal. A reduced isolation radius of $\Delta R=0.2$ has the effect of boosting the signal by $20 \%$. A similar answer was found for the background in ref. [40]; an understanding of the effect of this change in $\Delta R$ on the reducible backgrounds is needed before deciding whether this procedure can enhance the significance of the signal.

The chances of observing the $A^{0}$ would be improved if the value of $\beta$ were lower, since then the production would be raised. In Fig. 18 we show an example where low $\beta$ values are allowed by triviality. (This can be achieved, roughly speaking, when $|\alpha|$ is small, the top quark is light and the scalar masses moderate.) For this example, we chose the following values:

$$
\begin{array}{lll}
\text { Set F }: & M_{H}=350 \mathrm{GeV}, & M_{h}=150 \mathrm{GeV}, \quad M_{H^{ \pm}}=350 \mathrm{GeV}, \\
& M_{t}=120 \mathrm{GeV}, \quad \alpha=0^{\circ} .
\end{array}
$$

We notice that for $\beta \lesssim 30^{\circ}$ a highly significant signal is possible, at least for Model II. Recall, though, our earlier comment that such low $\beta$ values are in danger of being already ruled out by experiment. (Both the measurement of $B R(b \rightarrow s \gamma)$ and that of the $Z \rightarrow b \bar{b}$ decay width set lower bounds on $\beta$; for charged Higgs masses of a few hundred GeV the bound from the latter is the strongest [23].) If this example represents the 'corner' of parameter space where the prospects of detection of the $A^{0}$ in this mode are optimal, Set D (see (19)) of parameter values is such that no point in the $\left(M_{A^{0}}, \beta\right)$ plane gives a $5 \sigma$ signal in either luminosity.

Summarizing, we remark that the $l \gamma \gamma X$ mode may give a clear signal in only a narrow range of parameter space, due to the low $A^{0} t \bar{t}$ production rate, which, for masses below 
$150 \mathrm{GeV}$ approximately, is considerably smaller than the corresponding rate for the Standard Higgs. Consequently, this mode was found to be useful only if $\beta \lesssim 35^{\circ}$, a range that may easily be disallowed by triviality (or experiment). If the integrated luminosity at the LHC is $10 \mathrm{fb}^{-1}$, then the $A^{0}$ can be observed in this mode only in exceptional cases. Comparison with the inclusive two-photon mode reveals the latter, under the optimistic assumptions listed in Section 5, to be a more promising option. The $l \gamma \gamma X$ mode can be a useful independent check, but enough events can only be accumulated if $\beta$ is relatively small and the high luminosity option at the LHC is used.

\section{$7 \quad$ The $Z \gamma \gamma$ channel}

In Section 4 we observed that the branching fraction of the $A^{0}$ to the states containing other scalars $\left(Z h, Z H, W^{ \pm} H^{\mp}\right)$ is generally large, and suggested that these modes may prove valuable in the search for the $C P$-odd Higgs, particularly for large $M_{A^{0}}$ where the two-photon mode cannot be relied on. In this section we examine in detail the process

$$
A^{0} \rightarrow Z h \rightarrow l^{+} l^{-} \gamma \gamma
$$

where $l$ stands for an electron or a muon. A similar discussion applies to the mode $A^{0} \rightarrow Z H$. For definiteness, we shall keep $H$ heavy. Allowing for the possibility that $H$ is light enough to have an appreciable branching ratio to two photons is straightforward to implement, but would not add to our understanding of the phenomenology of the $A^{0}$.

The direct background to (28) comes primarily from the process

$$
q \bar{q} \rightarrow Z \gamma \gamma
$$

It was found to be small, as we show below, so we did not consider necessary to perform the calculation of gluon fusion into $Z \gamma \gamma$ (through the 'pentagon' diagram) which could in principle be comparable. There is another process that can interfere with (29), namely the standard production of a $C P$-even Higgs in association with a $Z$ :

$$
q \bar{q} \rightarrow Z^{\star} \rightarrow Z h(\rightarrow Z \gamma \gamma)
$$

This was also found to be very small, so we will neglect it in what follows. The calculation of (29) was done using spinor techniques [29]. The cross-section is singular when the photons are emitted along the beam axis or are soft. The following cuts were employed 
to exclude such photons and simulate a realistic situation:

$$
\begin{array}{llr}
\left|\eta^{l, \gamma}\right|<2.5 & p_{T}^{l, \gamma}>20 \mathrm{GeV} \\
\Delta R\left(\gamma_{1}, \gamma_{2}\right)>0.4 & , & \Delta R\left(l_{1}, l_{2}\right)>0.4 \\
\Delta R(l, \gamma)>0.4 & . &
\end{array}
$$

The isolation cut for photons and leptons has only a $5 \%$ effect on this calculation. We also included reconstruction efficiencies of $90 \%$ for each lepton and $85 \%$ for each photon after isolation cuts (see ref. [37).

Fig. 19a shows a plot of the number of $Z \gamma \gamma$ background events per GeV per LHC year (at a luminosity of $10^{34} \mathrm{~cm}^{-2} \mathrm{~s}^{-1}$ ) as a function of the $\gamma \gamma$ invariant mass. For an invariant mass in the range $40 \mathrm{GeV} \lesssim M_{\gamma \gamma} \lesssim 160 \mathrm{GeV}$ (the interesting mass range for a Higgs $h$ to decay to two photons), a $3 \%$ detector resolution will yield $\sim 2$ events of background. A similar conclusion is reached by looking at Fig. 19b, which plots events per $\mathrm{GeV}$ per LHC year versus the total final state invariant mass. For typical values of parameters that result in a signal of several events, the width of $A^{0}$ is a few $\mathrm{GeV}$. The background in such a bin is about 1 event. (The $A^{0}$ can be considerably broader at high mass, but there the $M_{Z \gamma \gamma}$ distribution dies out.) The $h$ and $A^{0}$ resonances will appear as bumps in the $M_{\gamma \gamma}$ and $M_{Z \gamma \gamma}$ distributions respectively, and it is the same events that will constitute both bumps. This is not the case with the background, however. The background events with a two-photon invariant mass $M_{\gamma \gamma}$ within $3 \%$ of $M_{h}$ will have a broad distribution in $M_{Z \gamma \gamma}$ and will not, in general, be concentrated in the vicinity of the $A^{0}$ resonance. This observation enables us to effectively eliminate the background. For example, Higgs masses of $M_{h}=100 \mathrm{GeV}, M_{A^{0}}=300 \mathrm{GeV}$ may typically give a signal of a few tens of events. Of the $\sim 2$ background events that have $M_{\gamma \gamma}$ inside a $3 \%$ window around $100 \mathrm{GeV}$, less than 0.1 lie within $5 \mathrm{GeV}$ of $M_{A^{0}}$. Hence it becomes plain that the background that simultaneously emulates both $h$ and $A^{0}$ is truly negligible.

QCD corrections to process (29) have not been calculated. We also did not estimate the size of reducible backgrounds from jet events $(Z \gamma j, Z j j)$. It is assumed that, with the efficiencies and rejection capabilities mentioned in Section 5 [37], these backgrounds will be brought to below the level of the direct $Z \gamma \gamma$ production. But in view of the arguments of the preceding paragraph, even if the reducible backgrounds were to dominate by as much as an order of magnitude over the irreducible $Z \gamma \gamma$ production, they would still pose no significant problems.

It is evident, therefore, that the observability of the $A^{0}$ in this mode depends only on whether a sufficient number of signal events can be accumulated. The magnitude of the light neutral Higgs branching ratio to two photons, $B R(h \rightarrow \gamma \gamma)$, is thus of crucial importance. This branching ratio has a complicated dependence on the various masses 
and angles. We will indicate very roughly what this dependence is so as to make the pattern of our results more intelligible.

A Higgs $h$ in the intermediate-mass range $40 \mathrm{GeV} \lesssim M_{h} \lesssim 160 \mathrm{GeV}$, decays mostly to a $b \bar{b}$ pair. Therefore, to a good approximation,

$$
B R(h \rightarrow \gamma \gamma) \approx \frac{\Gamma(h \rightarrow \gamma \gamma)}{\Gamma(h \rightarrow b \bar{b})}
$$

From the $h b \bar{b}$ couplings displayed in Table 1, it follows that

$$
\Gamma(h \rightarrow b \bar{b}) \sim \begin{cases}\cos ^{2} \alpha / \sin ^{2} \beta & (\text { Model I) } \\ \sin ^{2} \alpha / \cos ^{2} \beta & (\text { Model II })\end{cases}
$$

The partial width $\Gamma(h \rightarrow \gamma \gamma)$ is more complicated. The light neutral Higgs $h$ decays to two photons through a loop in which a $W$, an $H^{+}$or a charged fermion flows. For moderate values of $\beta$ the contribution of the $W$ is the largest, although, in some cases, the top quark loop is of comparable magnitude. The $h W W$ coupling (see Table 1 ) is proportional to $\sin (\beta-\alpha)$ so we may write, very roughly,

$$
B R(h \rightarrow \gamma \gamma) \sim \begin{cases}\sin ^{2}(\beta-\alpha) \sin ^{2} \beta / \cos ^{2} \alpha & (\text { Model I) } \\ \sin ^{2}(\beta-\alpha) \cos ^{2} \beta / \sin ^{2} \alpha & \text { (Model II) }\end{cases}
$$

provided none of the sines and cosines that appear in these formulae are too small. Another feature of the $\gamma \gamma$ branching ratio of the scalar $h$ is that it becomes largest for $M_{h} \approx$ 120-130 GeV and has a very soft dependence on $M_{H^{ \pm}}$and $M_{t}$.

Our prime motivation for considering this decay mode was to explore regions of parameter space where the two-photon channel gives a signal too faint to observe. In Fig. 20 we show $Z \gamma \gamma$ event contours for Set D of parameters (see (19)). More than 10 events are expected for points inside these contours. The solid curve (corresponding to an integrated luminosity of $L=10 \mathrm{fb}^{-1}$ ) can also be interpreted as a 100-event contour for $L=100 \mathrm{fb}^{-1}$. The branching ratio for the $Z$ decay into electrons or muons, as well as the kinematical cuts (31) and the lepton and photon reconstruction efficiencies, were included in the calculation of the signal. QCD corrections to the decays of $h$ were taken into account in a way similar to that described in Section 4 for the $A^{0}$. The large value of $|\alpha|$ (see (19)) results in a huge rate in the case of Model I, where, at $L=100 \mathrm{fb}^{-1}$, a clear signal is obtained for almost the entire parameter space depicted. For Model II the results are more modest, but comparison with Fig. 13 shows that, even in this case, the $Z \gamma \gamma$ signal

\footnotetext{
${ }^{8}$ In addition, the known corrections to the two-gluon mode [34] were also included.
} 
is observable in a region where the two-photon mode can hardly be helpful. Choosing $|\alpha|$ to be small would instead produce a large rate in Model II. This is illustrated in Fig. 21. The only difference relative to Set $\mathrm{D}$ is the value of $\alpha$ which in this example is $\alpha=0^{\circ}$. We shall refer to this choice as 'Set G'. Here too, the inclusive two-photon mode fails to give an observable signal for $A^{0}$ masses beyond the threshold for the $Z h$ decay. (We omit plots for this, however.)

In Section 5 we remarked that the $\gamma \gamma$ signal is weak for large $M_{A^{0}}$, when other channels open up, and for large $\beta$, when the production rate (as well as the two-photon branching ratio in Model II) is small. From (34) it follows, however, that, depending on $\alpha$ and the choice of Model, the light $C P$-even Higgs branching ratio to two photons may be enhanced at large $\beta$ and perhaps even compensate for the loss in production rate. An example where this happens is given in Fig. 22. The parameters belong to Set C, which represents the case where the $A^{0} Z h$ coupling is weak enough to allow a sizeable $\gamma \gamma$ branching ratio to persist up to the top threshold (see Fig. 12). Indeed, the $Z h$ mode can't give a good signal beyond this threshold either, but, at high luminosity, it covers some of the large- $\beta$ regions undetectable by the two-photon mode, while being able to confirm the latter for smaller $\beta$ and $M_{Z}+M_{h}<M_{A^{0}}<2 M_{t}$.

In order to convey an idea on the dependence of our results on $\alpha$, we present in Fig. 23 similar 10-event contours in the $(\alpha, \beta)$ space, now keeping $M_{A^{0}}$ fixed:

$$
\begin{aligned}
& \text { Set H : } \quad M_{H}=400 \mathrm{GeV}, \quad M_{h}=100 \mathrm{GeV}, \quad M_{H^{ \pm}}=350 \mathrm{GeV} \text {, } \\
& M_{A^{0}}=280 \mathrm{GeV}, \quad M_{t}=150 \mathrm{GeV} \text {. }
\end{aligned}
$$

The precise value of $M_{A^{0}}$ has no effect on these curves, as long as it is below the $W^{ \pm} H^{\mp}$ or $t \bar{t}$ threshold. The different behaviour of the two Models for large and small $|\alpha|$ is evident from the figure. We observe that large numbers of events are expected in a considerable region of parameter space. The only area left unexplored corresponds to $\alpha>0$ and large $\beta$.

The situation is not as rosy if $M_{A^{0}}>2 M_{t}$ or $M_{A^{0}}>M_{W}+M_{H^{ \pm}}$, in which case the branching ratio to $Z h$ is smaller. A clear signal of a few tens of events is then guaranteed in Model I (resp. Model II) only for large (resp. small) $|\alpha|$.

In all figures shown the value $M_{h}=100 \mathrm{GeV}$ was assumed. We remarked earlier that the branching ratio of $h$ to two photons increases with $M_{h}$ until approximately $M_{h} \approx 120 \mathrm{GeV}$ and then falls again. The corresponding $Z \gamma \gamma$ event rates would of course follow the same trend.

In the MSSM the decay sequence $A^{0} \rightarrow Z h \rightarrow l^{+} l^{-} \gamma \gamma$ discussed in this section has not been considered, presumably because it gives too low a rate. We have mentioned, in Section 5 , that the bias on $\tan \beta$ tends to suppress the $A^{0} Z h$ coupling in the kinematical 
region where the decay $A^{0} \rightarrow Z h$ is possible. Consequently, the branching ratio of this decay is only sizeable when $\tan \beta$ is not very large and $M_{Z}+M_{h}<M_{A^{0}}<2 M_{t}$ [10]. However, in this range, the branching ratio of $h$ to two photons does not exceed $10^{-3}$ (except for large $M_{t}$ ) [10]. In contrast, in the context of the non-supersymmetric Two-Doublet Model, it can reach the level of a few percent (and even higher in exceptional cases). The different behaviour can be traced to the fact that $\alpha$ is an independent parameter in the Two-Doublet Model and may assume values such as to enhance this branching ratio. The decay $A^{0} \rightarrow Z h$ in the MSSM has received some consideration in conjunction with the subsequent $\tau^{+} \tau^{-}$44] or $b \bar{b}$ [13 decays of the light Higgs $h$, with some encouraging results in the former case. The $\tau^{+} \tau^{-}$branching ratio of an intermediate-mass $h$ in the Two-Doublet Model, as well as in the MSSM, is about 10\%, almost independently of $\alpha$ and $\beta$. Consequently we may expect this mode to be helpful also in the Two-Doublet Model; in particular, it may enable us to explore the region for $\alpha>0$ where the $Z \gamma \gamma$ signal is weak. A detailed study of the signal and backgrounds at the LHC is needed in order to decide this issue.

To summarize, we have found that the process $A^{0} \rightarrow Z h \rightarrow l^{+} l^{-} \gamma \gamma$ provides an excellent way of simultaneously detecting the $A^{0}$ and the light $C P$-even scalar $h$, provided the latter is in the intermediate mass-region $40 \mathrm{GeV} \lesssim M_{h} \lesssim 160 \mathrm{GeV}$. This is true even at a yearly luminosity of $10 \mathrm{fb}^{-1}$ at the LHC. Tens or hundreds of signal events can be obtained over a negligible background in a fairly large region of parameter space, especially when $|\alpha|$ is large (in Model I) or small (in Model II). This mode only fares poorly when $\alpha$ is positive and moderate and $\beta$ large, or when the $t \bar{t}$ or $W^{ \pm} H^{\mp}$ channels are available for the decay of $A^{0}$. If the latter is true, detection is harder but not impossible.

The utility of the $Z h$ channel extends also to larger masses for the $C P$-even Higgs $h$. If $M_{h} \gtrsim 160 \mathrm{GeV}$, then $h$ can be detected in its 'gold-plated' decay mode $h \rightarrow Z Z$ or $Z Z^{\star} \rightarrow$ $l^{+} l^{-} l^{+} l^{-}$, with $l=e, \mu$. We have estimated that the process $A^{0} \rightarrow Z h$ followed by the above decay sequence may yield several events provided $M_{A^{0}}<2 M_{t}$. The last requirement shows that the kinematical region where this six-lepton signal can be observed is rather narrow, unless the top quark is relatively heavy $\left(M_{t} \gtrsim 150 \mathrm{GeV}\right)$. Nevertheless, this may be the only way of detecting the $C P$-odd scalar in this region of parameter space.

\section{Conclusions}

In this paper we examined the phenomenology of the $C P$-odd scalar $A^{0}$ of a two-Higgsdoublet model with an exact discrete symmetry. We explored the parameter space defined by the triviality bounds of ref. [6] in order to identify the regions where the $A^{0}$ can be detected at the LHC. We studied three different signals of the $A^{0}$. The inclusive two- 
photon mode can provide an observable signal if $\beta \lesssim 55-60^{\circ}$ and provided the decays of the $A^{0}$ to $t \bar{t}, W^{ \pm} H^{\mp}, Z h$ are kinematically forbidden or (in the case of $Z h$ ) suppressed. The lower end of the $A^{0}$ mass range where detection in this mode is possible depends on $\beta$ and is raised as $\beta$ increases. Models with small $\beta$ lead to larger signals because the production rate of $A^{0}$ is effectively proportional to $\cot ^{2} \beta$, at least for moderate values of $\beta$. This is also true for the associated $t \bar{t} A^{0}$ production, which was found capable of giving a detectable signal in the $l^{ \pm} \gamma \gamma X$ mode if $\beta \lesssim 35-40^{\circ}$ (and for roughly the same range of $M_{A^{0}}$ as in the inclusive two-photon case). However, this range is mostly disallowed by triviality bounds (as well as disfavoured by current experimental data). If the integrated luminosity at the LHC is $10 \mathrm{fb}^{-1}$, this mode can only be helpful in exceptional cases. In contrast, the process $A^{0} \rightarrow Z h$, followed by the two-photon decay of the light $C P$-even Higgs $h$, can provide a very clear signal for a substantial region of parameter space, as long as $h$ is in the so-called intermediate-mass range ( $40 \mathrm{GeV} \lesssim M_{h} \lesssim 160 \mathrm{GeV}$ ). (For heavier $h$, its decay to four leptons through a $Z$ pair may give rise to several events if the masses are such that $M_{Z}+M_{h}<M_{A^{0}}<2 M_{t}$.) This mode is largely complementary to the $\gamma \gamma$ mode since it covers regions of high $M_{A^{0}}$ as well as (in some instances) high $\beta$. Under certain circumstances, it may also confirm observation of the $A^{0}$ in the two-photon channel. Hundreds of events can be accumulated in this mode if $|\alpha|$ is large in Model I or small in Model II.

If $M_{A^{0}}>2 M_{t}$ or $M_{A^{0}}>M_{W}+M_{H^{ \pm}}$, then the branching ratio to $Z h$ is reduced and our ability to detect the $C P$-odd scalar in this mode is compromised. Although for some values of $\alpha$ and $\beta$ this mode would still be a prime option for the direct observation of $A^{0}$, it may be worthwhile investigating other processes, such as the $t \bar{t} A^{0}$ production with the subsequent decay of $A^{0}$ to $t \bar{t}$ [13]. Another region that, according to our conclusions, escapes observation at the LHC, is the low- $M_{A^{0}}$, large- $\beta$ region (essentially regardless of the values of the other parameters). It may be possible to explore part of this region at LEP-II, particularly since the disadvantage of insufficient production at large $\beta$ will not be present there.

It would be interesting to perform similar studies for the other scalars of the TwoDoublet Model, in order to determine how much of the parameter space of such models can be explored at future colliders. Work in this direction is under progress.

\section{Acknowledgements}

I wish to thank R. S. Chivukula for suggesting to me the project of the investigation of the phenomenology of two-doublet models and discussing it at all its stages, K. Lane and M. V. Ramana for very useful discussions and M. Golden, V. Koulovassilopoulos and M. V. Ramana for allowing me to use some of their FORTRAN routines. This work 
was supported in part under NSF contract PHY-9057173 and DOE contract DE-FG0291ER40676, and by funds from the Texas National Research Laboratory Commission under grant RGFY93-278.

\section{References}

[1] ALEPH Collaboration, Phys. Rep. 216 (1992) 253;

DELPHI Collaboration, P. Abreu et al., Nucl. Phys. B373 (1992) 3;

L3 Collaboration, O. Adriani et al., Phys. Lett. B303 (1993) 391;

OPAL Collaboration, M. Akrawy et al., Phys. Lett. B253 (1991) 511.

[2] M. Aizenman, Phys. Rev. Lett. 47 (1981) 1; Comm. Math. Phys. 86 (1982) 1;

J. Fröhlich, Nucl. Phys. B200 [FS4] (1982) 281;

A. D. Sokal, Ann. Inst. H. Poincaré A 37 (1982) 317.

[3] R. Dashen and H. Neuberger, Phys. Rev. Lett. 50 (1983) 1897.

[4] M. Lüscher and P. Weisz, Nucl. Phys. B318 (1989) 705;

J. Kuti, L. Lin and Y. Shen, Phys. Rev. Lett. 61 (1988) 678;

A. Hasenfratz, K. Jansen, C. B. Lang, T. Neuhaus and H. Yoneyama, Phys. Lett. B199 (1987) 531;

A. Hasenfratz, K. Jansen, J. Jersák, C. B. Lang, T. Neuhaus and H. Yoneyama, Nucl. Phys. B317 (1989) 81;

G. Bhanot, K. Bitar, U. M. Heller and H. Neuberger, Nucl. Phys. B353 (1991) 551.

[5] For example, D. Froidevaux, in Proceedings of the ECFA Large Hadron Collider Workshop, Aachen 1990, (G. Jarlskog and D. Rein, eds.), Vol. II, p. 444, and references therein.

[6] D. Kominis and R. S. Chivukula, Phys. Lett. B304 (1993) 152.

[7] GEM Technical Design Report; GEM TN-93-262, SSCL-SR-1219; Submitted by the GEM Collaboration to the Superconducting Super Collider Laboratory (April 30, 1993).

[8] W. J. Marciano and F. E. Paige, Phys. Rev. Lett. 66 (1991) 2433.

[9] J. F. Gunion, Phys. Lett. B261 (1991) 510.

[10] Z. Kunszt and F. Zwirner, Nucl. Phys. B385 (1992) 3. 
[11] V. Barger, M. S. Berger, A. L. Stange and R. J. N. Phillips, Phys. Rev. D 45 (1992) 4128 ;

J. F. Gunion, R. Bork, H. Haber and A. Seiden, Phys. Rev. D 46 (1992) 2040;

H. Baer, M. Bisset, D. Dicus, C. Kao and X. Tata, Phys. Rev. D 47 (1993) 1062.

[12] J. F. Gunion and L. Orr, Phys. Rev. D 46 (1992) 2052.

[13] J. F. Gunion, H. Haber and C. Kao, Phys. Rev. D 46 (1992) 2907.

[14] V. Barger, K. Cheung, R. J. N. Phillips and A. L. Stange, Phys. Rev. D 46 (1992) 4914.

[15] H. Baer, M. Bisset, C. Kao and X. Tata, Phys. Rev. D 46 (1992) 1067.

[16] S. L. Glashow and S. Weinberg, Phys. Rev. D 15 (1977) 1958.

[17] J. F. Gunion, H. E. Haber, G. L. Kane and S. Dawson, The Higgs hunter's guide, (Addison-Wesley, Reading, MA, 1990).

[18] M. Sher, Phys. Rep. 179 (1989) 273.

[19] J. F. Gunion and H. Haber, Nucl. Phys. B272 (1986) 1;

S. Bertolini, Nucl. Phys. B272 (1986) 77.

[20] C. -P. Yuan et al., preprint MSU 93/25 (hep-ph 9311226).

[21] R. Ammar et al., Phys. Rev. Lett. 71 (1993) 674.

[22] L. Rolandi and R. Tanaka, Proc. XXVI Int. Conf. on High Energy Physics, Dallas, 1992.

[23] A. J. Buras, M. Misiak, M. Münz and S. Pokorski, preprint MPI-Ph/93-77 hep-ph 9311345).

[24] G. Park, preprint CTP-TAMU-69/93 (hep-ph 9311207).

[25] H. M. Georgi, S. L. Glashow, M. E. Machacek and D. V. Nanopoulos, Phys. Rev. Lett. 40 (1978) 692.

[26] R. P. Kauffman and W. Schaffer, preprint BNL-49061 (hep-ph 9305279).

[27] E. Eichten, I. Hinchliffe, K. Lane and C. Quigg, Rev. Mod. Phys. 56 (1984) 579; 58 (1986) 1065 (E).

[28] D. Dicus and S. Willenbrock, Phys. Rev. D 39 (1989) 751. 
[29] R. Kleiss and W. J. Stirling, Nucl. Phys. B262 (1985) 235.

[30] D. J. Summers, Phys. Lett. B306 (1993) 129.

[31] J. F. Gunion, G. Gamberini and S. F. Novaes, Phys. Rev. D 38 (1988) 3481;

T. J. Weiler and T.-C. Yuan, Nucl. Phys. B318 (1989) 337.

[32] E. Braaten and J. Leveille, Phys. Rev. D 22 (1980) 715;

M. Drees and K. Hikasa, Phys. Lett. B240 (1990) 445; 262 (1991) 497 (E);

R. Kleiss, Z. Kunszt and W. J. Stirling, Phys. Lett. B253 (1991) 269.

[33] A. Djouadi, M. Spira and P. M. Zerwas, Phys. Lett. B311 (1993) 255.

[34] A. Djouadi, M. Spira and P. M. Zerwas, Phys. Lett. B264 (1991) 440.

[35] P. Aurenche et al., Z. Phys. C29 (1985) 459;

H. Baer and J. Owens, Phys. Lett. B205 (1988) 377;

B. Bailey, J. Owens and J. Ohnemus, Phys. Rev. D 46 (1992) 2018.

[36] P. Aurenche et al., in Proceedings of the ECFA Large Hadron Collider Workshop, Aachen 1990, (G. Jarlskog and D. Rein, eds.), Vol. II, p. 83.

[37] C. Seez et al., in Proceedings of the ECFA Large Hadron Collider Workshop, Aachen 1990, (G. Jarlskog and D. Rein, eds.), Vol. II, p. 474.

[38] CMS Collaboration, Expression of Interest, presented at the General Meeting on LHC Physics and Detectors, Evian-les-Bains, France, March 1992.

[39] Z. Kunszt, Nucl. Phys. B247 (1984) 339.

[40] Z. Kunszt, Z. Trócsányi and W. J. Stirling, Phys. Lett. B271 (1991) 247.

[41] A. Ballestrero and E. Maina, Phys. Lett. B268 (1991) 437.

[42] D. J. Summers, Phys. Lett. B277 (1992) 366.

[43] P. N. Harriman, A. D. Martin, W. J. Stirling and R. G. Roberts, Phys. Rev. D 42 (1990) 798.

[44] H. Baer, C. Kao and X. Tata, Phys. Lett. B303 (1993) 284. 


\section{Figure captions}

1. Production cross-section of the $C P$-odd Higgs $A^{0}$ through gluon fusion as a function of its mass for (a) Model I and (b) Model II. Results are shown for $\beta=15^{\circ}, 45^{\circ}, 70^{\circ}$ and $88^{\circ}$ and for $M_{t}=150 \mathrm{GeV}$.

2. Production cross-section of the $A^{0}$ through $b \bar{b}$ fusion as a function of its mass for (a) Model I and (b) Model II and for the same set of values of $\beta$ as in Fig. 1.

3. Cross-section for the associated $t \bar{t} A^{0}$ production as a function of the $A^{0}$ mass for (a) $M_{t}=120 \mathrm{GeV}$ and (b) $M_{t}=180 \mathrm{GeV}$ and for the same set of values of $\beta$ as in Fig. 1 . There is no distinction between Models I and II in this case. The corresponding cross-section for the Standard Higgs is also shown for comparison.

4. Total production cross-section of the $A^{0}$ as a function of its mass for (a) Model I and (b) Model II and for the same set of values of $\beta$ as in Fig. 1. The corresponding cross-section for the Standard Higgs (not including gauge boson fusion production) is also shown. The top quark mass is taken to be $150 \mathrm{GeV}$.

5. Branching ratios of the $A^{0}$ as a function of its mass for the following choices of parameters: (a) $M_{H}=400 \mathrm{GeV}, M_{h}=260 \mathrm{GeV}, M_{H^{ \pm}}=350 \mathrm{GeV}, M_{t}=120 \mathrm{GeV}, \alpha=$ $30^{\circ}, \beta=30^{\circ}$, Model I; (b) same for Model II; (c) same as (a) except $\beta=60^{\circ}$; (d) same as (b) except $\beta=60^{\circ}$.

6. Branching ratios of the $A^{0}$ as a function of its mass for the following choices of parameters: (a) $M_{H}=400 \mathrm{GeV}, M_{h}=100 \mathrm{GeV}, M_{H^{ \pm}}=350 \mathrm{GeV}, M_{t}=180 \mathrm{GeV}, \alpha=$ $30^{\circ}, \beta=40^{\circ}$, Model I; (b) same for Model II; (c) same as (a) except $\alpha=-45^{\circ}$; (d) same as (b) except $\alpha=-45^{\circ}$.

7. Production cross-section times branching ratio to two photons for the $A^{0}$ as a function of its mass for (a) Model I and (b) Model II and for the following set of parameters: $M_{H}=400 \mathrm{GeV}, M_{h}=260 \mathrm{GeV}, M_{H^{ \pm}}=350 \mathrm{GeV}, M_{t}=120 \mathrm{GeV}, \alpha=30^{\circ}$. Curves are shown for $\beta=15^{\circ}, 45^{\circ}, 70^{\circ}$ and $88^{\circ}$ as well as for the Standard Higgs.

8. Same as Fig. 7 but for the following values of parameters: $M_{H}=400 \mathrm{GeV}, M_{h}=$ $100 \mathrm{GeV}, M_{H^{ \pm}}=350 \mathrm{GeV}, M_{t}=180 \mathrm{GeV}, \alpha=30^{\circ}$.

9. Same as Fig. 7 but for the following values of parameters: $M_{H}=400 \mathrm{GeV}, M_{h}=$ $100 \mathrm{GeV}, M_{H^{ \pm}}=350 \mathrm{GeV}, M_{t}=180 \mathrm{GeV}, \alpha=-45^{\circ}$.

10. Significance contours for the inclusive two-photon signal in the region of the $\left(M_{A^{0}}, \beta\right)$ plane allowed by triviality for (a) Model I and (b) Model II. The other parameters 
are as in Fig. 7. Contours are shown for integrated luminosities of $10 \mathrm{fb}^{-1}$ and $100 \mathrm{fb}^{-1}$. The statistical significance is greater than $5 \sigma$ in the interior of these contours.

11. Same as Fig. 10 but for the set of parameters of Fig. 8.

12. Same as Fig. 10 but for the set of parameters of Fig. 9.

13. Same as Fig. 10 but for the following choice of parameters: $M_{H}=400 \mathrm{GeV}, M_{h}=$ $100 \mathrm{GeV}, M_{H^{ \pm}}=350 \mathrm{GeV}, M_{t}=180 \mathrm{GeV}, \alpha=-75^{\circ}$.

14. Associated $t \bar{t} A^{0}$ production cross-section times branching ratio to an $l^{ \pm} \gamma \gamma$ final state as a function of $M_{A^{0}}$. This branching ratio is the product of the $A^{0} \rightarrow \gamma \gamma$ branching fraction and the probability that at least one of the $t$ and $\bar{t}$ decays leptonically. Results are shown for (a) Model I and (b) Model II and for $\beta=15^{\circ}, 45^{\circ}, 70^{\circ}$ and $88^{\circ}$. The remaining parameters are as follows: $M_{H}=400 \mathrm{GeV}, M_{h}=260 \mathrm{GeV}, M_{H^{ \pm}}=$ $350 \mathrm{GeV}, M_{t}=120 \mathrm{GeV}, \alpha=30^{\circ}$. The corresponding quantity for the Standard Higgs is also plotted for comparison.

15. Same as Fig. 14 except now $M_{t}=180 \mathrm{GeV}$.

16. Significance contours for the $l \gamma \gamma X$ mode in the region of the $\left(M_{A^{0}}, \beta\right)$ plane allowed by triviality for (a) Model I and (b) Model II. The other parameters are as in Fig. 14. No point in the region depicted can give a $5 \sigma$ signal if a yearly luminosity of $10 \mathrm{fb}^{-1}$ is employed.

17. Same as Fig. 16, but for the set of parameters of Fig. 15.

18. Same as Fig. 16, but for the following values of parameters: $M_{H}=350 \mathrm{GeV}, M_{h}=$ $150 \mathrm{GeV}, M_{H^{ \pm}}=350 \mathrm{GeV}, M_{t}=120 \mathrm{GeV}, \alpha=0^{\circ}$.

19. Continuum $Z \gamma \gamma$ background events per $\mathrm{GeV}$ per $100 \mathrm{fb}^{-1}$ as a function of (a) the two-photon invariant mass $M_{\gamma \gamma}$ and (b) the total final state invariant mass $M_{Z \gamma \gamma}$.

20. 10-event contours for the $Z \gamma \gamma$ signal in the region of the $\left(M_{A^{0}}, \beta\right)$ plane allowed by triviality for (a) Model I and (b) Model II. The slice of parameter space shown corresponds to the following set of parameters: $M_{H}=400 \mathrm{GeV}, M_{h}=100 \mathrm{GeV}, M_{H^{ \pm}}=$ $350 \mathrm{GeV}, M_{t}=180 \mathrm{GeV}, \alpha=-75^{\circ}$. Contours are shown for luminosities of $10 \mathrm{fb}^{-1}$ and $100 \mathrm{fb}^{-1}$. More than 10 events are expected in the interior of these contours.

21. Same as Fig. 20 except now $\alpha=0^{\circ}$.

22. Same as Fig. 20 except now $\alpha=-45^{\circ}$. 
23. 10-event contours for the $Z \gamma \gamma$ signal in the region of the $(\alpha, \beta)$ plane allowed by triviality for (a) Model I and (b) Model II. The remaining parameters take the following values: $M_{H}=400 \mathrm{GeV}, M_{h}=100 \mathrm{GeV}, M_{H^{ \pm}}=350 \mathrm{GeV}, M_{t}=$ $150 \mathrm{GeV}, M_{A^{0}}=280 \mathrm{GeV}$. 
This figure "fig1-1.png" is available in "png" format from: http://arxiv.org/ps/hep-ph/9402339v1 
This figure "fig2-1.png" is available in "png" format from: http://arxiv.org/ps/hep-ph/9402339v1 
This figure "fig3-1.png" is available in "png" format from: http://arxiv.org/ps/hep-ph/9402339v1 
This figure "fig1-2.png" is available in "png" format from: http://arxiv.org/ps/hep-ph/9402339v1 
This figure "fig2-2.png" is available in "png" format from: http://arxiv.org/ps/hep-ph/9402339v1 
This figure "fig3-2.png" is available in "png" format from: http://arxiv.org/ps/hep-ph/9402339v1 
This figure "fig1-3.png" is available in "png" format from: http://arxiv.org/ps/hep-ph/9402339v1 
This figure "fig2-3.png" is available in "png" format from: http://arxiv.org/ps/hep-ph/9402339v1 
This figure "fig3-3.png" is available in "png" format from: http://arxiv.org/ps/hep-ph/9402339v1 
This figure "fig1-4.png" is available in "png" format from: http://arxiv.org/ps/hep-ph/9402339v1 
This figure "fig2-4.png" is available in "png" format from: http://arxiv.org/ps/hep-ph/9402339v1 
This figure "fig3-4.png" is available in "png" format from: http://arxiv.org/ps/hep-ph/9402339v1 